\title{
First confirmatory study on PTPRQ as an autosomal dominant non-syndromic hearing loss gene
}

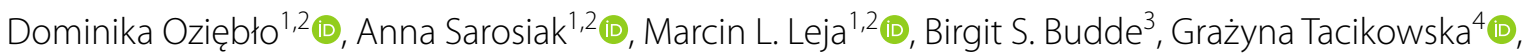

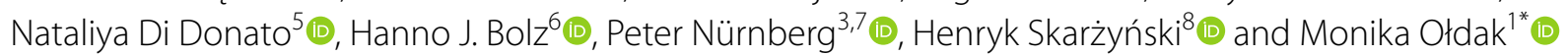

\begin{abstract}
Background: Biallelic PTPRQ pathogenic variants have been previously reported as causative for autosomal recessive non-syndromic hearing loss. In 2018 the first heterozygous PTPRQ variant has been implicated in the development of autosomal dominant non-syndromic hearing loss (ADNSHL) in a German family. The study presented the only, so far known, PTPRQ pathogenic variant (c.6881G $>$ A) in ADNSHL. It is located in the last PTPRQ coding exon and introduces a premature stop codon (p.Trp2294*).
\end{abstract}

Methods: A five-generation Polish family with ADNSHL was recruited for the study $(n=14)$. Thorough audiological, neurotological and imaging studies were carried out to precisely define the phenotype. Genomic DNA was isolated from peripheral blood samples or buccal swabs of available family members. Clinical exome sequencing was conducted for the proband. Family segregation analysis of the identified variants was performed using Sanger sequencing. Single nucleotide polymorphism array on DNA samples from the Polish and the original German family was used for genome-wide linkage analysis.

Results: Combining clinical exome sequencing and family segregation analysis, we have identified the same (NM_001145026.2:c.6881G>A, NP_001138498.1:p.Trp2294*) PTPRQ alteration in the Polish ADNSHL family. Using genome-wide linkage analysis, we found that the studied family and the original German family derive from a common ancestor. Deep phenotyping of the affected individuals showed that in contrast to the recessive form, the PTPRQ-related ADNSHL is not associated with vestibular dysfunction. In both families ADNSHL was progressive, affected mainly high frequencies and had a variable age of onset.

Conclusion: Our data provide the first confirmation of PTPRQ involvement in ADNSHL. The finding strongly reinforces the inclusion of $P T P R Q$ to the small set of genes leading to both autosomal recessive and dominant hearing loss.

Keywords: Hearing loss, PTPRQ, Pathogenic, Dominant, Next-generation sequencing, Linkage

\footnotetext{
*Correspondence: m.oldak@ifps.org.pl

1 Department of Genetics, World Hearing Center, Institute of Physiology and Pathology of Hearing, M. Mochnackiego 10, 02-042 Warsaw, Poland Full list of author information is available at the end of the article
} 


\section{Background}

Hearing loss (HL) is the most common disability of human senses. It is considered to have a monogenic origin in at least half of the individuals developing HL prior to speech acquisition. Similar data are not available for postlingual HL but it is noticeable that familial aggregation of later-onset, progressive HL often follows an autosomal dominant pattern of inheritance. Up to now, 46 genes involved in the development of autosomal dominant non-syndromic HL (ADNSHL) have been identified (https://hereditaryhearingloss.org; accessed 10/2019). New genes related to ADNSHL are still being discovered and many of the identified variants in known HL genes represent novel changes. This highlights a great genetic heterogeneity of ADNSHL $[1,2]$.

In 2018, Eisenberger et al. [3] proposed PTPRQ (MIM*603317) as a new candidate gene for ADNSHL. The conclusion was based on the identification of a novel heterozygous nonsense PTPRQ variant p.Trp2294* that cosegregated with progressive HL in a German family. $P T P R Q$ encodes protein tyrosine phosphatase receptor type Q, which catalyzes the dephosphorylation of phosphatidylinositol phosphates and displays a low activity against phosphotyrosine $[4,5]$. Expression of PTPRQ in the inner ear is confined to hair bundles in the cochlea and in the vestibule and distributed depending on the hair cell type or its location, primarily in the basal region of hair bundles. In the cochlea, high PTPRQ protein level is detected in its basal turn responding to high frequency sound [6]. The main PTPRQ isoform in the inner ear contains a long extracellular domain, a short hydrophobic transmembrane region and an intracellular domain with a single phosphatase catalytic site [7].

The p.Trp2294* variant is predicted to introduce a premature termination codon (PTC) shortening the PTPRQ protein at the $\mathrm{C}$-terminus by the last six amino acid residues. However, PTPRQ expression at the mRNA level remains unaffected, indicating that the mutant transcript escapes nonsense-mediated decay and supporting the hypothesis that the truncated PTPRQ protein is produced [3]. Performing additional functional studies to confirm the mutant variant mechanism of action was hampered by the large size of the PTPRQ protein and small difference in size between the wild-type and mutant protein. Since the initial report no additional data have been provided to unequivocally confirm $P T P R Q$ as an ADNSHL gene.

Our study provides novel family-based evidence, confirming the pathogenic role of PTPRQ in ADNSHL. Based on the results of clinical exome sequencing, we identified the same $P T P R Q$ variant as Eisenberger et al. in a new five-generation Polish ADNSHL family and found that both families have a common ancestor. Our strong genetic and clinical data constitute an ultimate confirmation for the causative role of PTPRQ in ADNSHL.

\section{Materials and methods Study subjects}

A five-generation Polish family with progressive, highfrequency ADNSHL was recruited for the study (Fig. 1a). For linkage analysis, four family members of the previously reported German family were also included [3].

\section{Clinical evaluation}

Auditory function of all participating family members was evaluated by pure tone audiometry. In the proband (IV.2) and her son (V.1) the examination was extended by impedance audiometry, transient evoked and distortion product otoacoustic emissions (TEOAE, DPOAE) and auditory brainstem responses (ABRs). Function of the vestibular system was assessed by cervical and ocular vestibular evoked myogenic potentials (cVEMP, oVEMP) with a $500-\mathrm{Hz}$ tone burst at $97 \mathrm{dBnHL}$ and FitzgeraldHallpike bithermal caloric stimulation with videonystagmography [8]. Anatomy of the auditory system was visualized by temporal bone computed tomography (Siemens CT Definition AS, Germany).

\section{Next-generation sequencing of clinical exome and Sanger sequencing}

Genomic DNA of the 14 family members (8 affected and 6 unaffected) was isolated from blood samples with a standard procedure or from buccal swabs using Maxwell FSC DNA IQ Casework Kit (Promega, Madison, Wisconsin, USA) according to the manufacturer's protocol. Next-generation sequencing of clinical exome was performed on proband's DNA using TruSight One Sequencing Kit (Illumina, San Diego, California, USA) and a MiSeq platform (Illumina) with $2 \times 150$ bp pairedend reads according to the manufacturer's protocol. All bioinformatic analyses were done as described previously [8]. Variants potentially related to ADNSHL in the family were selected based on the prediction scores from computational algorithms (including CADD, LRT, FATHHM, MutationTaster, PolyPhen-2, SIFT) and their frequency in population databases (including gnomAD, ESP6500, UK10K). Sanger sequencing using BigDye Terminator cycle sequencing kit v.3.1 (Applied Biosystems, Foster City, CA, USA) and 3500xL Genetic Analyzer (Applied Biosystems) was performed to confirm the presence and segregation of the identified PTPRQ and TMC1 (MIM*606706) variants in all studied family members.

\section{Genome-wide linkage analysis}

Genome-wide linkage analysis with DNA samples of the Polish (IV.1, IV.2, V.1, V.2) and German (DEIII.1, DEIII.2, 


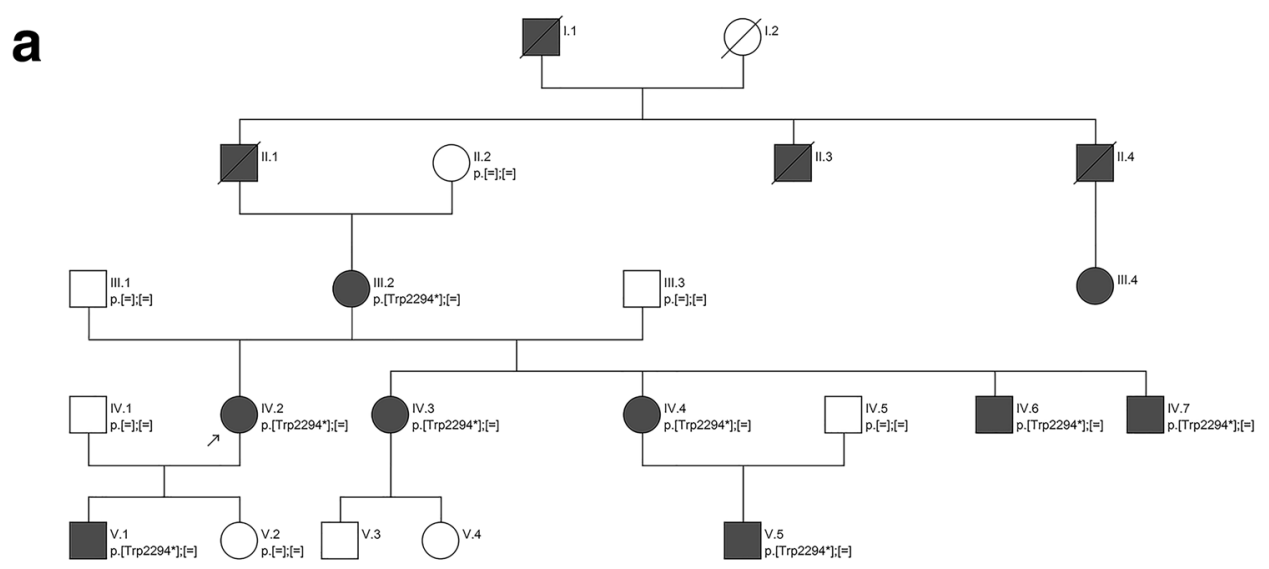

b

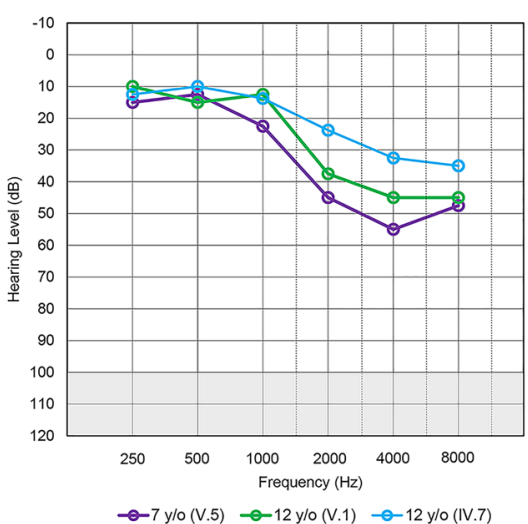

d

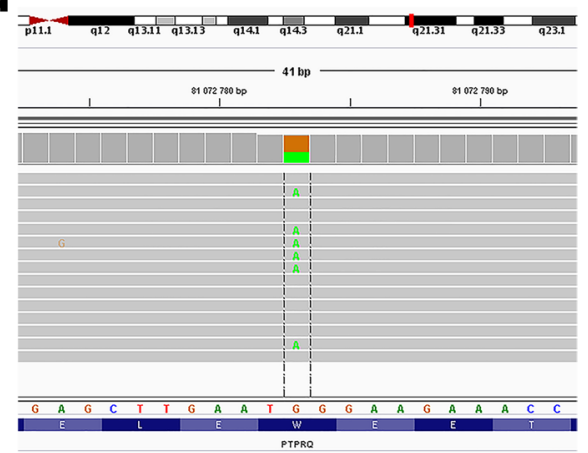

C

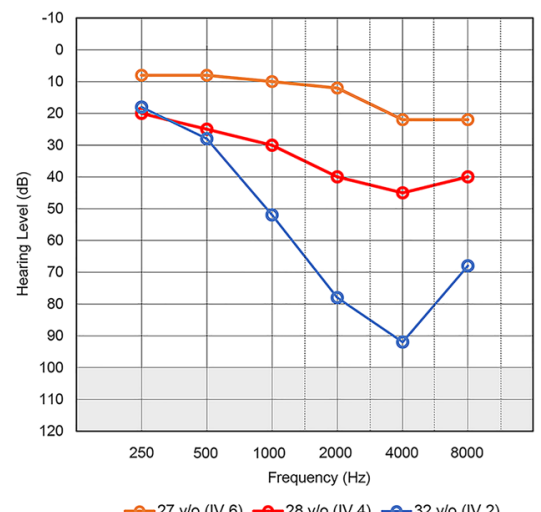

e

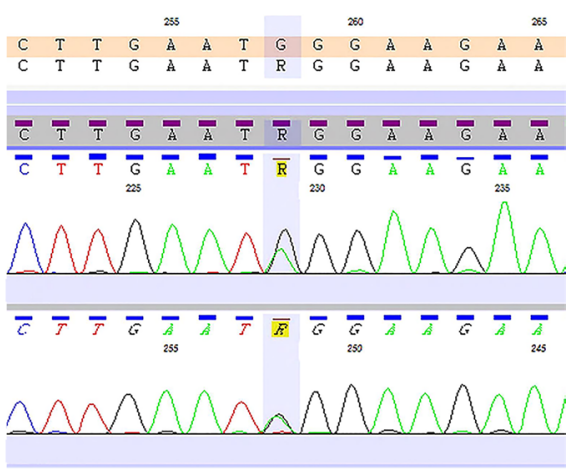

Fig. 1 Identification of a heterozygous c.6881G>A PTPRQ pathogenic variant in a 5-generation ADNSHL family. a Family pedigree showing a typical autosomal dominant mode of inheritance and cosegregation of the PTPRQ variant with HL. The proband is marked with an arrow. Affected individuals are indicated by black symbols, unaffected individuals are indicated by open symbols, diagonal line denotes deceased family members. b, $\mathbf{c}$ Pure tone audiometry of selected family members at a similar age between 7 and $12 \mathrm{y} / \mathrm{o}$ (b) and 27-32 y/o (c) showing varying degrees of hearing loss. "O" symbols denote a mean binaural values of air conduction thresholds. $\mathbf{d}$ Clinical exome sequencing results visualized with the Integrative Genomic Viewer software illustrating the presence of a heterozygous guanine to adenine transition (c.6881G>A) (green letters) localized within exon 45. of the PTPRQ gene and resulting in a premature stop codon (p.Trp2294*). e The corresponding electropherogram from Sanger sequencing 
DEIV.2 and DEIV.4 according to [3]) families was carried out using the Axiom Precision Medicine Research Array (Thermo Fisher Scientific, Waltham, MA, USA). Genotypes were called by the Axiom Analysis Suite v4.0 and processed as previously reported [3]. Multipoint LOD scores were calculated and haplotypes reconstructed with MERLIN [9].

\section{Results}

Affected family members from the examined Polish family suffered from bilateral, progressive, high-frequency HL with an onset from 4 to 27 years of age. The degree of HL showed a high intrafamilial variability ranging from an almost normal hearing status to severe HL (Table 1). Detailed audiological evaluation revealed severe HL in the proband (IV.2, $32 \mathrm{y} / \mathrm{o}$ ) and mild to moderate HL in her son (V.1, $12 \mathrm{y} / \mathrm{o})$ at mid and high frequencies that was accompanied by tinnitus. At low frequencies hearing thresholds were within the normal range (Fig. 1a-c). In both individuals, tympanograms were normal and stapedial reflexes were present with ipsi- and contralateral stimulation with the exception of high frequencies where their thresholds were increased or absent. Otoacoustic emissions were bilaterally absent in the proband and recorded in her son up to $1500 \mathrm{~Hz}$ in the right and up to $1000 \mathrm{~Hz}$ in the left ear. Corresponding with the HL degree, ABRs recordings were lacking waves I and III, while wave $\mathrm{V}$ latencies were normal. Combined results of the objective hearing measurements showed bilateral hearing impairment originating from a defective cochlear component of the auditory system. Neurotological examinations showed cVEMP and oVEMP responses and results of bi-thermal caloric irrigation within normal limits. There were no malformations of the auditory system on temporal bone imaging (Additional file 1: Figures S1-S7).

Molecular genetic testing showed the presence of a heterozygous NM_001145026.2:c.6881G>A variant in the PTPRQ gene, which fully segregated with ADNSHL in the studied family (Fig. 1a, d, e). The identified variant is located in the last coding exon of PTPRQ and introduces a PTC (NP_001138498.1:p.Trp2294*). The PTPRQ variant has not been reported in population databases but was detected in a single previously published German ADNSHL family [3]. Based on European descent and the close geographic proximity, a common ancestor was assumed. An affected and an unaffected offspring together with their parents were genotyped on a genomewide single nucleotide polymorphism (SNP) array. The region surrounding the shared $P T P R Q$ variant was analyzed for linkage in each family. The subsequent reconstruction of haplotypes revealed that a SNP haplotype spanning $306 \mathrm{~kb}$ (from rs1358476 to rs7978990) is shared by both families (Additional file 2: Figure S8).

In addition to the PTPRQ variant identified in the proband, we have found six other genetic alterations in HL genes that had population allele frequency below 0.001 and were present in protein coding regions. Considering the mode of $\mathrm{HL}$ inheritance and literature data, we have selected the TMC1 NM_138691.2:c.1705A>G variant for family segregation study and found that it was present in normal hearing individuals and absent in some affected family members [10]. Its presence did not correspond with a more severe HL phenotype within the family.

\section{Discussion}

Our report is the first confirmatory study on the causative role of PTPRQ in ADNSHL development. Here, we have identified eight previously unreported individuals suffering from progressive, high-frequency ADNSHL caused by the c.6881G $>$ A PTPRQ nonsense variant (p.Trp2294*), which represents the first and so far the only PTPRQ pathogenic variant involved in ADNSHL. Up to now, it was reported exclusively by Eisenberger et al. [3] who, after detailed examination of a German family, proposed PTPRQ as a novel ADNSHL candidate gene (assigned at the DFNA73 locus 12q21.31). Their assumption was based on the results of exome sequencing, supported by linkage analysis, extended sequencing of the alternatively spliced exons and in silico predicted, potential $P T P R Q$ coding regions.

In their paper, Eisenberger et al. have listed variants in genes reportedly associated with hearing impairment and variants located in the mapped candidate region encompassing $P T P R Q$ that were included in the final analysis of potential ADNSHL pathogenic variants in the studied family. We have carefully compared the high throughput sequencing data of the German proband with the sequencing results of our proband and found that the majority of variants were not present in our patient except for variants within $S Y T 1$ and TMTC2 genes flanking PTPRQ on chromosome 12. Current data from the gnomAD database showed that both variants have a high population frequency $(0.0023$ and 0.0097, respectively) and are present in homozygous state in 2 and 43 individuals, respectively. This allowed to unequivocally exclude both genes from further consideration.

To verify whether the detected PTPRQ variant is located within the same inherited chromosomal region or represents an independent event that occurred by chance at the same position in both families, genomewide linkage analysis was performed. After testing four family members from each family, we found that the 


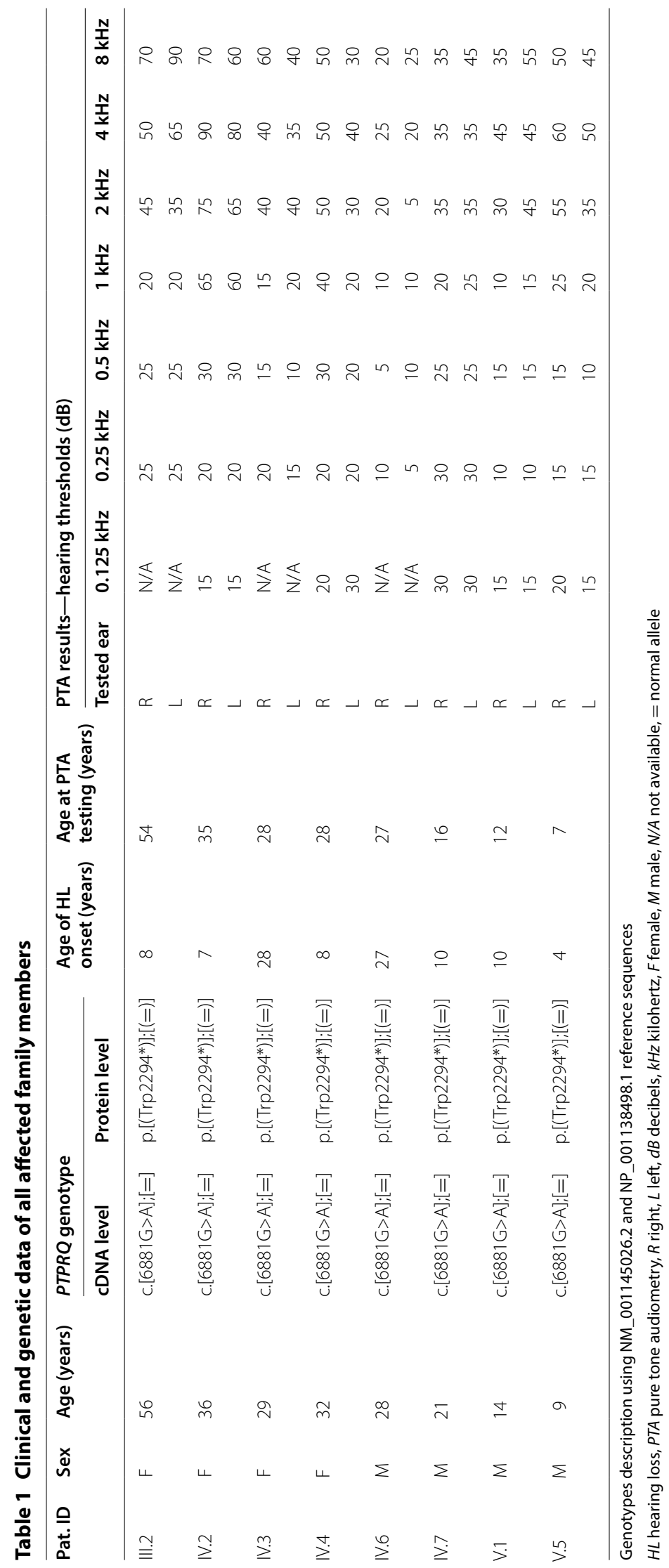


PTPRQ c.6881G >A variant is present within a haplotype shared by the affected individuals from both families. It is located almost in the middle of a common chromosomal region containing the distal half of the PTPRQ gene together with the following $3^{\prime}$ region. The data have clearly demonstrated that the c.6881G $>$ A containing $P T P R Q$ chromosomal region did not undergo recombination and HL patients in both families share copies of the same part of the ancestral PTPRQ haplotype.

Here, for the first time we have performed deep phenotyping of the affected individuals and found that the defect caused by the pathogenic PTPRQ p.Trp2294* variant is restricted to the cochlear component of the auditory system. In contrast to individuals with $P T P R Q$ recessive variants who suffer from vestibular dysfunction diagnosed in infancy or early childhood, our patients do not develop a vestibular disorder $[11,12]$.

The exact function of the PTPRQ protein remains poorly understood. Its large extracellular part forms shaft connectors most probably involved in proper spacing of stereocilia and maintaining their stability. The intracellular fragment containing an active enzymatic site was found to regulate the level of phosphatidylinositol phosphates. It has been also proposed that the PTPRQ C-terminus interacts with a protein complex containing MYO6, RDX, TPRN and CLIC5 [13], encoded by known HL genes. Proper function of the protein complex is required to maintain typical localization of PTPRQ at the base of stereocilia. In MYO6- and CLIC5-deficient mice, PTPRQ cannot be properly compartmentalized and it dissociates to the upper parts of the shafts leading to destabilization of membrane-cytoskeletal attachments, membrane lifting and consequent fusing or loosing of stereocilia [13-16].

The p.Trp2294* variant terminates the intracellular region of the PTPRQ protein after 30 amino acid residues following its catalytic site. It is the only PTPRQ pathogenic variant reported so far that results in a shortened PTPRQ protein with a preserved catalytic domain. One may speculate that the truncated PTPRQ may not be able to properly interact with its molecular partners and may diffuse and disturb the gradient of phosphatidylinositol phosphates along the shafts in a gain of function mechanism. Another possibility is that the pathologically shortened protein could disturb the function of the normal PTPRQ copy. It may exert a dominant negative effect and compete with the normal PTPRQ protein for binding of its molecular partners or co-participate in the multi-protein complex formation. As a consequence, the defective PTPRQ protein would weaken the protein complex and lead to insufficient stereocilia resistance to mechanical forces. This could modulate HL age of onset and severity which could explain the large intra- and interfamilial difference in this regard. In both studied families ADNSHL begins and progresses mainly at high frequencies adopting gradually a "ski-slope" curve on an audiogram, which is typical for HL induced by environmental factors.

\section{Conclusions}

In summary, PTPRQ is better known as a recessive HL gene $[11,12,17-21]$. The present study strongly reinforces its inclusion to the small set of genes leading to both autosomal recessive and dominant hearing loss. Our data provide the first independent confirmation of PTPRQ causative role in ADNSHL.

\section{Supplementary information}

Supplementary information accompanies this paper at https://doi. org/10.1186/s12967-019-2099-5.

Additional file 1: Figure S1-S7. Results of proband's audiological evaluation.

Additional file 2: Figure S8. Linkage analysis for chromosome 12 in individuals from German (DEIV.2, DEIV.4) and Polish (V.1, V.2) families.

\begin{abstract}
Abbreviations
ABRs: auditory brainstem responses; ADNSHL: autosomal dominant non-syndromic hearing loss; cVEMP: cervical vestibular evoked myogenic potentials; DPOAE: distortion product otoacoustic emissions; HL: hearing loss; OVEMP: ocular vestibular evoked myogenic potentials; PTC: premature termination codon; SNP: single nucleotide polymorphism; TEOAE: transient evoked otoacoustic emissions.
\end{abstract}

\section{Acknowledgements}

Not applicable.

\section{Authors' contributions}

$\mathrm{MO}, \mathrm{DO}$ analyzed the data and wrote the manuscript; $\mathrm{DO}, \mathrm{AS}, \mathrm{MLL}, \mathrm{BB}, \mathrm{PN}$ performed genotyping and analyzed the data; MO, DO, GT, HJB, NDD, HS participated in phenotyping and clinical data collection; DO, AS, BB prepared figures. All authors read and approved the final manuscript.

\section{Funding}

This study was supported by National Science Centre Grant No. 2016/22/E/ NZ5/00470.

\section{Ethics approval and consent to participate}

The study was approved by the bioethics committee at the Institute of Physiology and Pathology of Hearing and performed according to the Declaration of Helsinki. Written informed consent was obtained from each participant.

\section{Consent for publication}

A consent for publication has been obtained.

Availability of data and materials

Please contact author for data requests.

\section{Competing interests}

The authors declare that they have no competing interests.

\section{Author details}

${ }^{1}$ Department of Genetics, World Hearing Center, Institute of Physiology and Pathology of Hearing, M. Mochnackiego 10, 02-042 Warsaw, Poland.

2 Postgraduate School of Molecular Medicine, Medical University of Warsaw, Warsaw, Poland. ${ }^{3}$ Cologne Center for Genomics (CCG), University of Cologne, Cologne, Germany. ${ }^{4}$ Department of Otoneurology, Institute of Physiology 
and Pathology of Hearing, Warsaw, Poland. ${ }^{5}$ Institute for Clinical Genetics, TU Dresden, Dresden, Germany. ${ }^{6}$ Senckenberg Zentrum für Humangenetik, Frankfurt am Main, Germany. ${ }^{7}$ Center for Molecular Medicine Cologne (CMMC), University Hospital Cologne, Cologne, Germany. ${ }^{8}$ Department of Oto-Rhino-Laryngology Surgery Clinic, Institute of Physiology and Pathology of Hearing, Warsaw, Poland.

Received: 16 July 2019 Accepted: 15 October 2019

Published online: 26 October 2019

\section{References}

1. Hereditary hearing loss and deafness overview. https://www.ncbi.nlm. nih.gov/books/NBK1434/.

2. Hereditary hearing loss homepage. https://hereditaryhearingloss.org.

3. Eisenberger T, Di Donato N, Decker C, Delle Vedove A, Neuhaus C, Nurnberg G, Toliat M, Nurnberg P, Murbe D, Bolz HJ. A C-terminal nonsense mutation links PTPRQ with autosomal-dominant hearing loss, DFNA73. Genet Med. 2018;20:614-21.

4. Seifert RA, Coats SA, Oganesian A, Wright MB, Dishmon M, Booth CJ, Johnson RJ, Alpers CE, Bowen-Pope DF. PTPRQ is a novel phosphatidylinositol phosphatase that can be expressed as a cytoplasmic protein or as a subcellularly localized receptor-like protein. Exp Cell Res. 2003;287:374-86.

5. Yu KR, Kim YJ, Jung SK, Ku B, Park H, Cho SY, Jung H, Chung SJ, Bae KH, Lee SC, et al. Structural basis for the dephosphorylating activity of PTPRQ towards phosphatidylinositide substrates. Acta Crystallogr D Biol Crystallogr. 2013;69:1522-9.

6. Goodyear RJ, Legan PK, Wright MB, Marcotti W, Oganesian A, Coats SA, Booth CJ, Kros CJ, Seifert RA, Bowen-Pope DF, Richardson GP. A receptorlike inositol lipid phosphatase is required for the maturation of developing cochlear hair bundles. J Neurosci. 2003;23:9208-19.

7. Wright MB, Hugo C, Seifert R, Disteche CM, Bowen-Pope DF. Proliferating and migrating mesangial cells responding to injury express a novel receptor protein-tyrosine phosphatase in experimental mesangial proliferative glomerulonephritis. J Biol Chem. 1998;273:23929-37.

8. Oldak M, Ozieblo D, Pollak A, Stepniak I, Lazniewski M, Lechowicz U, Kochanek K, Furmanek M, Tacikowska G, Plewczynski D, et al. Novel neuro-audiological findings and further evidence for TWNK involvement in Perrault syndrome. J Transl Med. 2017;15:25.

9. Abecasis GR, Cherny SS, Cookson WO, Cardon LR. Merlin-rapid analysis of dense genetic maps using sparse gene flow trees. Nat Genet. 2002;30:97-101.

10. Kurima K, Peters LM, Yang Y, Riazuddin S, Ahmed ZM, Naz S, Arnaud D, Drury S, Mo J, Makishima T, et al. Dominant and recessive deafness caused by mutations of a novel gene, TMC1, required for cochlear haircell function. Nat Genet. 2002;30:277-84.
11. Schraders M, Oostrik J, Huygen PL, Strom TM, van Wijk E, Kunst HP, Hoefsloot LH, Cremers CW, Admiraal RJ, Kremer H. Mutations in PTPRQ are a cause of autosomal-recessive nonsyndromic hearing impairment DFNB84 and associated with vestibular dysfunction. Am J Hum Genet. 2010;86:604-10.

12. Sakuma N, Moteki H, Azaiez H, Booth KT, Takahashi M, Arai Y, Shearer AE, Sloan CM, Nishio SY, Kolbe DL, et al. Novel PTPRQ mutations identified in three congenital hearing loss patients with various types of hearing loss. Ann Otol Rhinol Laryngol. 2015;124(Suppl 1):184S-92S.

13. Salles FT, Andrade LR, Tanda S, Grati M, Plona KL, Gagnon LH, Johnson KR, Kachar B, Berryman MA. CLIC5 stabilizes membrane-actin filament linkages at the base of hair cell stereocilia in a molecular complex with radixin, taperin, and myosin VI. Cytoskeleton (Hoboken). 2014;71:61-78.

14. Goodyear RJ, Jones SM, Sharifi L, Forge A, Richardson GP. Hair bundle defects and loss of function in the vestibular end organs of mice lacking the receptor-like inositol lipid phosphatase PTPRQ. J Neurosci. 2012;32:2762-72.

15. SelfT, Sobe T, Copeland NG, Jenkins NA, Avraham KB, Steel KP. Role of myosin VI in the differentiation of cochlear hair cells. Dev Biol. 1999;214:331-41.

16. Sakaguchi H, Tokita J, Naoz M, Bowen-Pope D, Gov NS, Kachar B. Dynamic compartmentalization of protein tyrosine phosphatase receptor $\mathrm{Q}$ at the proximal end of stereocilia: implication of myosin VI-based transport. Cell Motil Cytoskeleton. 2008;65:528-38.

17. Wu X, Wang S, Chen S, Wen YY, Liu B, Xie W, Li D, Liu L, Huang X, Sun Y, Kong WJ. Autosomal recessive congenital sensorineural hearing loss due to a novel compound heterozygous PTPRQ mutation in a Chinese family. Neural Plast. 2018;2018:9425725.

18. Talebi F, Ghanbari Mardasi F, Mohammadi Asl J, Tizno S, Najafvand Zadeh M. Identification of novel PTPRQ and MYO1A mutations in an Iranian pedigree with autosomal recessive hearing loss. Cell J. 2018;20:127-31.

19. Gao X, Su Y, Chen YL, Han MY, Yuan YY, Xu JC, Xin F, Zhang MG, Huang SS, Wang GJ, et al. Identification of two novel compound heterozygous PTPRQ mutations associated with autosomal recessive hearing loss in a Chinese family. PLOS ONE. 2015;10:e0124757.

20. Sang Q, Mei H, Kuermanhan A, Feng R, Guo L, Qu R, Xu Y, Li H, Jin L, He L, Wang L. Identification of a novel compound heterozygous mutation in PTPRQ in a DFNB84 family with prelingual sensorineural hearing impairment. Mol Genet Genom. 2015;290:1135-9.

21. Shahin H, Rahil M, Abu Rayan A, Avraham KB, King MC, Kanaan M, Walsh T. Nonsense mutation of the stereociliar membrane protein gene PTPRQ in human hearing loss DFNB84. J Med Genet. 2010;47:643-5.

\section{Publisher's Note}

Springer Nature remains neutral with regard to jurisdictional claims in published maps and institutional affiliations.

Ready to submit your research? Choose BMC and benefit from:

- fast, convenient online submission

- thorough peer review by experienced researchers in your field

- rapid publication on acceptance

- support for research data, including large and complex data types

- gold Open Access which fosters wider collaboration and increased citations

- maximum visibility for your research: over 100M website views per year

At BMC, research is always in progress.

Learn more biomedcentral.com/submissions 